\title{
Eye movements during long-term pictorial recall
}

\author{
Corinna S. Martarelli $\cdot$ Fred W. Mast
}

Received: 15 November 2011/ Accepted: 2 May 2012/Published online: 19 May 2012

(C) Springer-Verlag 2012

\begin{abstract}
We investigated eye movements during longterm pictorial recall. Participants performed a perceptual encoding task, in which they memorized 16 stimuli that were displayed in different areas on a computer screen. After the encoding phase the participants had to recall and visualize the images and answer to specific questions about visual details of the stimuli. One week later the participants repeated the pictorial recall task. Interestingly, not only in the immediate recall task but also 1 week later participants looked longer at the areas where the stimuli were encoded. The major contribution of this study is that memory for pictorial objects, including their spatial location, is stable and robust over time.
\end{abstract}

\section{Introduction}

Eye movements are involved in the processing of visual information but still relatively little is known about their role in cognitive tasks when there is no obvious reason to move one's eyes. It has been shown that the previous spatial position of absent objects can influence eye movements during recall and during mental imagery in adult participants (Altmann, 2004; Brandt \& Stark, 1997; Laeng \& Teodorescu, 2002; Richardson \& Spivey, 2000; Spivey \& Geng, 2001) and in children (Martarelli \& Mast, 2011; Richardson \& Kirkham, 2004). Johansson, Holsanova, and Holmqvist (2006), for example, instructed adult participants to first view and then imagine a complex picture or to create a mental image from a verbal description. Their

C. S. Martarelli $(\bowtie)$. F. W. Mast

Department of Psychology, University of Bern, Muesmattstrasse 45, CH-3000 Bern 9, Switzerland e-mail: corinna.martarelli@psy.unibe.ch results show that eye movements reflected the objects' positions while participants recalled both visually and verbally encoded stimuli. Such findings suggest that specific eye movements are generated when participants recall information from memory. Whereas previous studies addressed eye movements during short-term recall, little is known about whether eye movements are still present when the information is recalled later, for example 1 week after encoding. This study aims to investigate eye movements during long-term pictorial recall of seen objects by testing adult participants with a procedure including a perceptual encoding task followed by pictorial recall tasks, not only immediately after stimulus presentation but also 1 week later.

More than 40 years ago, Shepard (1967, Experiment 3) found that after inspection of 612 photographs the average performance in a recognition task was nearly $100 \%$. More recently, Hollingworth (2005) studied how natural scenes are stored in long-term visual memory by means of a change detection task at different delays ranging from $200 \mathrm{~ms}$ to $24 \mathrm{~h}$ after encoding. Since performance was above chance level 1 day after encoding he argued for the robustness of long-term memory for visual details. The present work extends Hollingworth's findings on tokenand orientation-change detection to long-term pictorial recall, including analyses of eye movements. If after 1 week, participants still look at blank spaces where they previously encoded the stimuli, we can conclude that not only visual details of objects such as their orientation or form but also their spatial position on the screen is stored in long-term memory.

Bourlon, Oliviero, Wattiez, Pouget, and Bartolomeo (2011) asked participants to visualize a map of France and to judge whether towns are situated left or right of Paris. They found that participants moved their eyes in the 
corresponding directions as if they actually saw the map. Humphrey and Underwood (2008) investigated eye movements when photographs of real-world scenes were visualized immediately after encoding but also $48 \mathrm{~h}$ later. They found similar eye movements during immediate imagery and delayed imagery (after 2 days). Whereas Humphrey and Underwood (2008) focused on the comparison between recognition and imagery of real-world scenes, we used pictorial objects located in different areas of a computer screen (the location is in our study irrelevant to the task). Furthermore, we extended the delay from $48 \mathrm{~h}$ to 1 week. Increasing the time between encoding and recall can be relevant with respect to system consolidation taking place over a more extended period of time, predominantly during sleep (Diekelmann \& Born, 2010; Frankland \& Bontempi, 2005). Future research will be needed to more thoroughly investigate how spatial location is consolidated along with the whole memory trace in long-term memory.

In addition to study long-term pictorial recall we manipulated eye position during recall. There has been early research manipulating eye movements that yielded contradictory results (Hale \& Simpson, 1971; Janssen \& Nodine, 1974). More recent studies by Richardson and Spivey (2000) and Laeng and Teodorescu (2002) also came to opposite conclusions. Richardson and Spivey (2000) manipulated eye movements during encoding and they found that eye movements occurred during auditory recall even then when participants could not use eye movements during encoding. Richardson and Spivey's (2000) results suggest that eye movements during retrieval are launched by abstract spatial representations. They concluded that encoding relies on attention shifts and not on eye movements per se. Laeng and Teodorescu (2002), however, found an improvement in memory performance when participants were free to move their eyes during visual encoding and later recall compared to when maintaining central fixation during recall. This finding implies that the eyes play a functional role when imagining objects. More recently, Johansson, Holsanova, Dewhurst, and Holmqvist (2011) carried out four experiments manipulating eye movements (central fixation) both during encoding and during retrieval. They found that eye movements play a functional role during retrieval but not during encoding. The authors conclude that eye movements assist the visual representation of spatial locations.

In this study, we manipulated eye position during pictorial recall both toward locations where the stimuli were encoded and toward non-corresponding locations. If eye position plays a functional role during recall, participants are expected to be less accurate when forced to look at noncorresponding areas compared to the original locations where they actually encoded the stimuli. It would rather speak against a reenactment of the scanpath during mental imagery if it turned out that the manipulation of eye movements does not lead to an impairment of accuracy.

In essence, we pursue two aims: first, we want to investigate eye movements to blank regions and long-term pictorial recall and second, we examine how eye movements relate to recall accuracy under eye-position manipulations.

\section{Methods}

\section{Participants}

Fifteen adults participated in the experiment. The results are based on thirteen participants (11 female and 2 male, aged between 20 and 42 years). The data from two adults had to be excluded because of inaccurate calibration values. All participants were naïve about the purpose of the experiment and had normal or corrected-to-normal visual acuity.

\section{Apparatus}

Eye movements were recorded using the iView X RED tracking system (SensoMotoric Instruments, Teltow, Germany). Data were registered with a sampling rate of $50 \mathrm{~Hz}$, a spatial resolution of $0.1^{\circ}$ and a gaze position accuracy of $0.5^{\circ}$. The eye-tracking device is contact free and determines the gaze on the image by combining the cornea reflex with the pupil location, via an infrared light sensitive video camera. The stimuli were presented on a 17 -in. screen using Experiment Center Software and eye data were recorded with I-View X Software, both developed by SensoMotoric Instruments.

\section{Stimuli}

The screen (17 in.) was divided by the vertical and horizontal midlines into four equally sized areas of interest (AOIs). During the perceptual encoding task colored stimuli appeared in one of the four areas (e.g., a parrot or an angel). There was an equal number of stimuli in each quadrant and the sequence of quadrants was random. The images were photographs modified with Adobe Photoshop and they covered on average a visual angle of $11.35^{\circ}$ (vertical) and $5.75^{\circ}$ (horizontal). See Appendix for a list of all the stimuli used in this study.

During the first and the last pictorial recall tasks (Fig. 1 represents a schematic illustration of the experimental procedure) the screen was white (participants were free to move their eyes), whereas during the pictorial recall task with eye-position manipulation a red rectangular frame highlighted one of the four AOIs (participants had to keep 
their gaze inside the area surrounded by the red rectangle). Based on Kosslyn, Thompson, and Ganis' (2006) visual buffer model, we divided the pictorial recall tasks into an image generation phase and an image inspection phase. During the image generation phase the participants had to recall and visualize as vividly as possible one of the previously seen pictures. Then, during the subsequent image inspection phase they had to respond to a detailed question about the picture (e.g., has the parrot blue wings?). All questions were specific to the stimuli and the participants responded by indicating "yes" or "no". Yes and no responses were equal in number and counterbalanced between tasks. The questions could not be answered by simply referring to conceptual knowledge about the stimuli (e.g., what one knows about parrots in general). The questions were recorded with a single speaker (AVI files).

\section{Procedure}

The experiment (see Fig. 1) consisted of two sessions 1 week apart. During the first session participants performed a perceptual encoding task, and two pictorial recall tasks: a short-term pictorial recall task and a pictorial recall task with eye-position manipulation. One week later they repeated the pictorial recall task. All the participants were tested individually and they sat $.5 \mathrm{~m}$ away from the computer screen.

Before each session, a nine-point calibration was conducted. The perceptual encoding task was composed of 16 visual stimuli each associated with an auditory stimulus (for example "This is a parrot") presented for $5 \mathrm{~s}$. After each stimulus presentation the screen became white and the participant recalled and visualized the stimulus (image

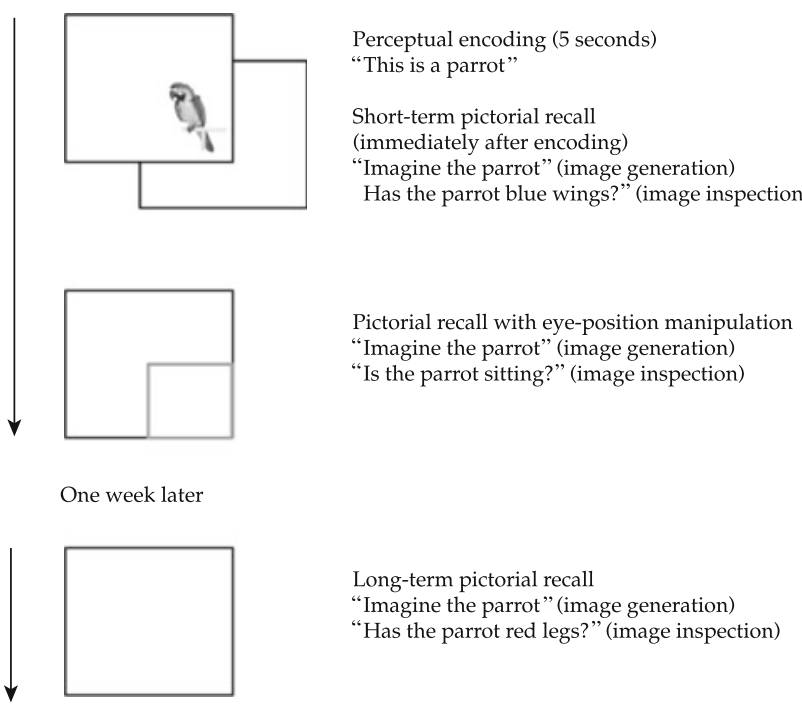

Fig. 1 A schematic representation of the temporal order of the experiment including stimuli used during the different tasks generation phase). Once they had generated the mental image they informed the experimenter by saying "ok", they instantly heard a specific question (auditory file) and made their response by pressing a button on the keyboard (image inspection phase). The short-term pictorial recall task allowed for controlling how well participants were able to encode the stimuli (short-term memory). After a brief delay the second pictorial recall task began and it now included eye-position manipulation. The minimum duration between short-term pictorial recall and pictorial recall with eye-position manipulation was $2 \mathrm{~min}$. This was the minimum time necessary to introduce the next task. In addition, the first image to recall in the task with eyeposition manipulation never corresponded to the last image participants had to recall in the short-term pictorial recall. We manipulated eye position toward AOIs where the stimuli were encoded and toward non-corresponding AOIs. For half of the stimuli eye position was manipulated to the corresponding AOI (i.e., the area where the stimulus was displayed during perceptual encoding) and for the other half of the stimuli eye position was manipulated to the noncorresponding AOI (two for each of the four non-corresponding AOIs). Participants were instructed to keep their gaze inside the area surrounded by a red rectangle. At the same time they had to first remember and visualize the previously seen stimulus (image generation phase) and then answer to a specific question (image inspection phase).

Even though the first experimental session was scheduled so that participants could come back exactly 7 days later, they were not aware that they will perform the same pictorial recall task again. Furthermore, the questions were new and differed from the questions asked during the preceding pictorial recall tasks. The questions were presented in random order.

\section{Results}

Fixations times

Eye movements were analyzed in the period starting from the onset of the auditory signal until the participant responded. The analyses were based on fixations and calculated using Be-Gaze software, SensoMotoric Instruments. Fixations were detected when the sum of the dispersion of the gaze stream on the $x$ and $y$ axes was below 100 pixels and when the duration exceeded $80 \mathrm{~ms}$. Saccades were analyzed by subtracting fixations and blink events from the original gaze stream. Fixation times below $100 \mathrm{~ms}$ were removed from the analyses. Fixation times of the three tasks were analyzed for correct trials only (there were too few incorrect trials for analyzing them 
separately). The four quadrants were defined as AOIs and we compared the time spent in the AOIs in which the pictures were displayed with the mean time spent fixating one of the non-corresponding AOIs with paired-samples $t$ tests and reported Cohen's (1988) $d$ measure of effect size. To avoid an overestimation of effect size, the measure was calculated from the original standard deviations (Dunlop, Cortina, Vaslow, \& Burke, 1996). To the extent that percentages allow for comparisons, we additionally report the percentage of time spent in the corresponding AOIs (chance level $25 \%$ ).

During the perceptual encoding task participants spent on average per trial 3,759 ms (SEM 99) fixating the AOI where the stimulus was displayed and $52 \mathrm{~ms}$ (SEM 6) fixating one of the non-corresponding AOIs, $t(12)=37.31$, $p<.001, d=11.06$, paired $t$ test; they spent $96 \%$ of the total time looking at the stimulus, and thus, they followed the instructions and understood the task. Immediately after each stimulus presentation, participants recalled the stimulus they just saw so that we could assess short-term memory performance. This task-as the other pictorial recall tasks - was divided in an image generation phase and an image inspection phase. During the image generation phase participants spent on average per trial $4,949 \mathrm{~ms}$ (SEM 469) fixating the corresponding AOI and $359 \mathrm{~ms}$ (SEM 103) fixating one of the non-corresponding AOIs, $t(12)=8.85, p<.001, d=3.48$, paired $t$ test; they spent $82 \%$ of the total time looking at the corresponding area. During the image inspection phase participants spent on average 3,165 ms (SEM 271) fixating the corresponding AOI and $340 \mathrm{~ms}$ (SEM 85) fixating one of the non-corresponding AOIs, $t(12)=8.73, p<.001, d=4.22$, paired $t$ test; they spent $76 \%$ of the total looking time at the corresponding AOI. The difference between the percentages of time spent in the corresponding area during the image generation and the image inspection phases did not reach statistical significance, $t(12)=1.64, p=.127$, $d=1.52$, paired $t$ test. Both during the image generation and the image inspection phase participants spent more time in the AOIs where they previously saw the stimuli even though they were free to move their eyes.

The analysis of the pictorial recall task with eye-position manipulation shows that participants kept their gaze in the area surrounded by the red rectangle both during the image generation phase and the image inspection phase: during the image generation phase they spent on average per trial $5,000 \mathrm{~ms}$ (SEM 444) in the areas surrounded by the red rectangle and $100 \mathrm{~ms}$ (SEM 13) in one of the non-corresponding areas, $t(12)=11.07, p<.001, d=3.15$, paired $t$ test (this is $94 \%$ of the total time) and during the image inspection phase they spent 4,569 ms (SEM 347) in the area surrounded with the red rectangle and $39 \mathrm{~ms}$ (SEM 15 ) in one of the non-corresponding areas, $t(12)=12.98$, $p<.001, d=3.78$, paired $t$ test (this is $97 \%$ of the time). Thus, in both phases they correctly understood and followed the instructions.

Analyses of the long-term pictorial recall task reveal that during the image generation phase participants spent on average 2,439 ms (SEM 496) fixating the corresponding AOI and 1,468 ms (SEM 181) fixating one of the noncorresponding AOIs, $t(12)=1.85, p=.088, d=.85$, paired $t$ test; they spent $34 \%$ of the total time looking in the AOI where the stimulus was encoded. During the image inspection phase of the long-term pictorial recall task participants spent on average per trial 2,333 ms (SEM 248) fixating the corresponding AOI and $840 \mathrm{~ms}$ (SEM 158) fixating one of the three non-corresponding AOIs, $t(12)=4.03, p=.002, d=4.59$, paired $t$ test, in percentages they spent $52 \%$ of the time in the corresponding AOI. Participants spent more time in the corresponding AOIs during the image inspection phase compared to the image generation phase, $t(12)=4.23, p=.001, d=1.7$, paired $t$ test.

We analyzed eye data of the long-term pictorial recall task according to the manipulation to the corresponding AOIs versus to the non-corresponding AOIs. Table 1 indicates that the eye-position manipulation did not influence eye fixations 1 week later; during the image inspection phase, participants spent more time in the AOI where they previously encoded the stimuli, regardless of where they looked during recall with eye-position manipulation.

When analyzing trials with manipulation to the noncorresponding AOIs participants spent $50 \%$ of the time in the corresponding AOI during the inspection phase of the long-term pictorial recall task. In addition to this, we can further divide the time spent in the non-corresponding areas in time spent in the cued area (the area where they looked at during manipulation) and time spent in one of the two uncued areas (mean time). A GLM analysis with a within-subjects factor area revealed a significant effect of the factor area, $F(2,206)=20.37, p<.001$, partial $\eta^{2}=.16$, indicating that participants spent more time in the corresponding area $(M=2,236 \mathrm{~ms}$, SEM 215), whereas there was no difference between the time spent in the cued area $(M=727 \mathrm{~ms}$, SEM 142) and the other two uncued areas $(M=1,208 \mathrm{~ms}$, SEM 160); pairwise comparisons with Bonferroni adjustment.

We additionally compared the short-term and the longterm pictorial recall tasks with paired $t$ tests. During the image generation phase participants spent $82 \%$ (SEM 4) of the time in the corresponding area during the short-term pictorial recall task and $34 \%$ (SEM 4) of the time in the long-term pictorial recall task, $t(12)=8.23, p<.001$, $\mathrm{d}=123.26$, paired $t$ test. During the image inspection phase participants spent $76 \%$ (SEM 5) of the time in the 
Table 1 Percent of time spent in the corresponding AOIs (where the stimuli where displayed previously) during the long-term pictorial recall task (separated for the image generation and the image inspection phases) according to the eye-position manipulation

\begin{tabular}{|c|c|c|}
\hline $\begin{array}{l}\text { Time spent in the } \\
\text { corresponding AOIs }\end{array}$ & $\begin{array}{l}\text { Image } \\
\text { generation } \\
\text { phase }\end{array}$ & $\begin{array}{l}\text { Image } \\
\text { inspection } \\
\text { phase }\end{array}$ \\
\hline $\begin{array}{l}\text { Manipulation to the } \\
\text { corresponding AOIs }\end{array}$ & $\begin{array}{l}34 \%(\text { SEM } 4 \%) \\
t(12)=2.079 \\
p=.06\end{array}$ & $\begin{array}{l}53 \%(\text { SEM } 8 \%) \\
t(12)=3.655 \\
p=.003\end{array}$ \\
\hline $\begin{array}{l}\text { Manipulation to the } \\
\text { non-corresponding AOIs }\end{array}$ & $\begin{array}{l}35 \%(\text { SEM } 4 \%) \\
t(12)=2.286 \\
p=.041\end{array}$ & $\begin{array}{l}50 \%(\text { SEM } 8 \%) \\
t(12)=3.016 \\
p=.011\end{array}$ \\
\hline Long-term pictorial recall & $\begin{array}{l}34 \%(\text { SEM } 4 \%) \\
t(12)=2.142 \\
p=.053\end{array}$ & $\begin{array}{l}52 \%(\text { SEM } 8 \%) \\
t(12)=3.669 \\
p=.003\end{array}$ \\
\hline
\end{tabular}

One-sample $t$ tests were computed to compare the percentages of time with chance level of $25 \%$ of the total time (four areas)

corresponding area during the short-term pictorial recall task and $52 \%$ (SEM 7) of the time in the long-term pictorial recall task, $t(12)=3.48, p=.005, \mathrm{~d}=3.63$, paired $t$ test. The analyses show that both during the image generation phase and the image inspection phase the effect of the corresponding area was stronger in the short-term pictorial recall task compared to the long-term pictorial recall task.

\section{Accuracy}

Participants were able to reliably solve all the tasks: they were correct in $89.9 \%$ (SEM $1.8 \%$ ) of the trials in the short-term pictorial recall task, in $81.6 \%$ (SEM $1.7 \%$ ) of the trials in the pictorial recall task with eye-position manipulation and in $80.3 \%$ (SEM $2.2 \%$ ) of the trials in the long-term pictorial recall task. The questions were matched for difficulty based on pretesting prior to the experiment. A GLM analysis with a within-subjects factor task (Mauchly's test showed that the condition of sphericity was met $\chi^{2}(2)=1.36, p=.505$ ) revealed a significant effect of the factor task, $F(2,24)=11.05, p<.001$, partial $\eta^{2}=.48$, indicating that participants were significantly better in the short-term pictorial recall task $(p<.04)$, whereas there was no difference between the pictorial recall task with eye-position manipulation and the pictorial recall task 1 week later (pairwise comparisons with Bonferroni adjustment). We carried out subsequent analyses by separating accuracy according to the eyeposition manipulation (corresponding vs. non-corresponding AOI's). None of the differences reached statistical significance $(p>.294$, pairwise comparisons with Bonferroni adjustment); participants were correct in $76.6 \%$
(SEM 2.4\%) of the trials with eye manipulations to the corresponding AOIs and in $88.4 \%$ (SEM 3.3\%) of the trials with eye manipulations to the non-corresponding AOIs. During the long-term pictorial recall task participants were correct in $77.8 \%$ (SEM $2.8 \%$ ) of the trials when eye position was manipulated to the corresponding AOIs 1 week earlier and in $86.5 \%$ (SEM $4.1 \%$ ) of the trials when eye position was manipulated to the non-corresponding AOIs 1 week earlier.

\section{Discussion}

Not only immediately after stimulus presentation, but also 1 week later, participants made specific eye movements when recalling pictorial information from memory. Even though participants were not explicitly asked to recall the locations (none of the participants guessed the hypothesis of the study), they stored the spatial information along with other visual properties in long-term memory.

In the long-term pictorial recall task participants' eyes fixated more often the areas where the stimuli were previously encoded, and thus, we conclude that the spatial information during pictorial recall is stored in long-term memory; eye movements are therefore not a temporary phenomenon that can only be observed shortly after encoding. This finding extends a previous study by Hollingworth (2005) in that not only visual features such as form or orientation but also the spatial position during encoding is stored in long-term memory. The effect of the corresponding area was stronger in the short-term pictorial recall task when compared to the long-term pictorial recall task. This finding was not unexpected because the stimuli and their location were still available in working memory immediately after presentation. In the long-term memory task, the effect of the corresponding area was stronger during the image inspection phase compared to the image generation phase. This is possibly due to the fact that it is only during the image inspection phase that the participants had to retrieve specific information from memory.

Manipulating eye position did not influence eye fixations during recall 1 week later, showing that the encoding of the spatial information cannot be disrupted easily. Our findings suggest that eye movements are part of the same mental representation, which also includes visual and semantic properties of the encoded stimuli (Ferreira, Apel, \& Henderson, 2008; Richardson, Altmann, Spivey, \& Hoover, 2009). Recall from memory when, for example, responding to a question during the image inspection phase activates the whole memory trace, including eye movements to the corresponding location.

As expected, participants were highly accurate during the short-term pictorial recall task $(89.9 \%)$. This ensured 
that they were able to properly encode the stimuli. They were slightly less accurate in the subsequent pictorial recall tasks (81.6 and $80.3 \%)$. Interestingly, there is no decrease in performance. It is possible that during the second pictorial recall task the information has already been stored in long-term memory. Another conceivable explanation is that the task with eye-position manipulation was more difficult; indeed, participants had to visualize the stimuli and answer specific questions while keeping their gaze inside the areas surrounded by the red rectangle. However, eye-position manipulation did not affect performance: participants performed equally well when they had to look where the stimulus was previously encoded or to a noncorresponding area on the screen. Hence, looking at the corresponding area did not facilitate task performance. This result is in line with Richardson and Spivey (2000) who found no improvement while participants revisited previously occupied locations. However, the result contradicts previous findings by Laeng and Teodorescu (2002) who showed a decrease in recall performance when participants kept their gaze on a fixation point. It has to be noted, however, that the eye fixation manipulations did not match entirely. Whereas Laeng and Teodorescu's participants fixated a cross (central fixation), our participants were free to move their eyes within predefined areas during recall. The advantage of our method is that there is no difference in working memory load. We have used this type of manipulation because having to maintain central fixation while imagining a previously stored image can by itself act like a distraction and thus influence task performance (Mast \& Kosslyn, 2002). Our results with eye-position manipulation suggest that the absolute spatial position per se is not crucial for accurate recall. However, before concluding that eye movements do not play a functional role during recall, further research is needed to investigate whether eye-position manipulation at yet other phases (e.g., during long-term pictorial recall) or with an increased number of repetitions can influence recall accuracy. It is possible that eye position does not affect performance when the image has been memorized recently, whereas it could play a role in recall performance 1 week later after a more extended consolidation phase.

Spivey and Geng (2001) showed that participants look more often to the previously occupied areas when the visual context is rich $(3 \times 3$ grid) as opposed to when the context is poor (frame instead of a grid). In our study, we decided to keep the visual context as minimal as possible (white screen) and, consequently, we provided no external cue, which could have possibly served as visual index. Nevertheless, participants spent more time in the areas where the stimuli were displayed previously. Hence, it appears unlikely that eye movements serve the sole purpose to spatially index external features (O'Regan, 1992; Pylyshyn, 2001, 2002, 2003).

To recapitulate, the present study suggests that participants' eye fixations during pictorial recall reflect the locations of where the stimuli were previously encoded both during short-term and long-term pictorial recall. Participants showed memory effects for the location even though they were neither asked to memorize nor to recall the position of the stimuli. We can therefore conclude that the spatial position during encoding is stored along with the whole memory trace, which integrates spatial, visual and semantic properties. We found no facilitation of task performance when the eyes were at the correct location. The present results suggest that memory for pictorial objects, including their spatial location, is robust and stable over time.

Acknowledgments This research was supported by the Swiss National Science Foundation, PDFM1-114406, Pro*Doc (awarded to FM). We thank all participants who took part in the study and Lilla Gurtner for her help during data collection.

\section{Appendix}

List of the 16 auditory labels (translations) used during the tasks.

Child, Cook, Man, Guitarist, Tennis player, Hindu Deity, Angel, Robot, Fox, Parrot, Sheep, Squirrel, Dog, Giraffe, Goat, Centaur.

\section{References}

Altmann, G. T. (2004). Language-mediated eye movements in the absence of a visual world: The 'blank screen paradigm'. Cognition, 93(2), B79-B87.

Bourlon, C., Oliviero, B., Wattiez, N., Pouget, P., \& Bartolomeo, P. (2011). Visual mental imagery: What the head's eye tells the mind's eye. Brain Research, 1367, 287-297.

Brandt, S. A., \& Stark, L. W. (1997). Spontaneous eye movements during visual imagery reflect the content of the visual scene. Journal of Cognitive Neuroscience, 9(1), 27-38.

Cohen, J. (1988). Statistical power analysis for the behavioral sciences (2nd ed.). Hillsdale, NJ: L. Erlbaum Associates.

Diekelmann, S., \& Born, J. (2010). The memory function of sleep. Nature Reviews Neuroscience, 11(2), 114-126.

Dunlop, W. P., Cortina, J. M., Vaslow, J. B., \& Burke, M. J. (1996). Meta-analysis of experiments with matched groups or repeated measures designs. Psychological Methods, 1(2), 170-177.

Ferreira, F., Apel, J., \& Henderson, J. M. (2008). Taking a new look at looking at nothing. Trends in Cognitive Sciences, 12(11), 405-410.

Frankland, P. W., \& Bontempi, B. (2005). The organization of recent and remote memories. Nature Reviews Neuroscience, 6(2), 119-130. 
Hale, S. M., \& Simpson, H. M. (1971). Effects of eye movements on rate of discovery and vividness of visual images. Perception \& Psychophysics, 9(2B), 242-246.

Hollingworth, A. (2005). The relationship between online visual representation of a scene and long-term scene memory. Journal of Experimental Psychology. Learning, Memory, and Cognition, 31(3), 396-411.

Humphrey, K., \& Underwood, G. (2008). Fixation sequences in imagery and in recognition during the processing of pictures of real-world scenes. Journal of Eye Movement Research, 2(2), $1-15$.

Janssen, W. H., \& Nodine, C. F. (1974). Eye-movements and visualimagery in free-recall. Acta Psychologica, 38(4), 267-276.

Johansson, R., Holsanova, J., Dewhurst, R., \& Holmqvist, K. (2011). Eye movements during scene recollection have a functional role, but they are not reinstatements of those produced during encoding. Journal of Experimental Psychology: Human Perception and Performance (Advance online publication).

Johansson, R., Holsanova, J., \& Holmqvist, K. (2006). Pictures and spoken descriptions elicit similar eye movements during mental imagery, both in light and in complete darkness. Cognitive Science, 30, 1053-1079.

Kosslyn, S. M., Thompson, W. L., \& Ganis, G. (2006). The case for mental imagery. New York: Oxford University Press.

Laeng, B., \& Teodorescu, D.-S. (2002). Eye scanpaths during visual imagery reenact those of perception of the same visual scene. Cognitive Science, 26, 207-231.

Martarelli, C. S., \& Mast, F. W. (2011). Preschool children's eyemovements during pictorial recall. British Journal of Developmental Psychology, 29, 425-436.
Mast, F. W., \& Kosslyn, S. M. (2002). Eye movements during visual mental imagery. Trends in Cognitive Sciences, 6(7), 271-272.

O'Regan, J. K. (1992). Solving the "real" mysteries of visual perception: The world as an outside memory. Canadian Journal of Psychology, 46(3), 461-488.

Pylyshyn, Z. W. (2001). Visual indexes, preconceptual objects, and situated vision. Cognition, 80(1-2), 127-158.

Pylyshyn, Z. W. (2002). Mental imagery: In search of a theory. Behavioral and Brain Sciences, 25(2), 157-238.

Pylyshyn, Z. W. (2003). Seeing and visualizing: It's not what you think. Cambridge: Mit Press.

Richardson, D. C., Altmann, G. T. M., Spivey, M. J., \& Hoover, M. A. (2009). Much ado about eye movements to nothing: A response to Ferreira et al.: Taking a new look at looking at nothing. Trends in Cognitive Sciences, 13(6), 235-236.

Richardson, D. C., \& Kirkham, N. Z. (2004). Multimodal events and moving locations: Eye movements of adults and 6-month-olds reveal dynamic spatial indexing. Journal of Experimental Psychology: General, 133(1), 46-62.

Richardson, D. C., \& Spivey, M. J. (2000). Representation, space and Hollywood squares: Looking at things that aren't there anymore. Cognition, 76(3), 269-295.

Shepard, R. N. (1967). Recognition memory for words, sentences, and pictures. Journal of Verbal Learning and Verbal Behavior, 6(1), $156-163$.

Spivey, M. J., \& Geng, J. J. (2001). Oculomotor mechanisms activated by imagery and memory: Eye movements to absent objects. Psychological Research, 65(4), 235-241. 